\title{
Sustainable Consumption with an Essential Exhaustible Resource Re-Examined
}

\author{
Juan Sesmero*, Lilyan Fulginiti \\ Purdue University, West Lafayette, USA \\ Email: "jsesmero@purdue.edu
}

Received 16 April 2016; accepted 5 June 2016; published 8 June 2016

Copyright (C) 2016 by authors and Scientific Research Publishing Inc.

This work is licensed under the Creative Commons Attribution International License (CC BY). http://creativecommons.org/licenses/by/4.0/

\section{c) (i) Open Access}

\begin{abstract}
This study derives conditions for existence of a positive sustainable consumption in an economy with an essential exhaustible resource. It does so by approximating technology with a variable elasticity of substitution production function, instead of the constant elasticity of substitution specification widely assumed in previous studies. This approach permits examination of the robustness of results previously derived in the literature to key technological assumptions. It also generates new insights regarding the role of substitutability and technical progress on existence. We find that a capital-resource elasticity of substitution greater than one is sufficient for existence even when the resource is strictly essential; a situation precluded by constant elasticity of substitution specifications. Under an elasticity of substitution lower than one, existence can still be attained (in contrast to the constant elasticity of substitution case) but only through capital-augmenting technical progress. Hicks-neutral technical progress is neither necessary nor sufficient for existence. A sufficiently high resource-augmenting technical progress thwarts existence of a positive sustainable consumption.
\end{abstract}

\section{Keywords}

Sustainable Consumption, Essential Exhaustible Resource, Variable Elasticity of Substitution, Non-Neutral Technical Progress

\section{Introduction}

This paper examines the role of input substitutability and technical progress on the existence of positive sustainable consumption in an economy with an essential exhaustible resource. The framework used in the literature to study the existence issue (e.g. [1]-[5]) is known as the Dasgupta-Heal-Solow-Stiglitz (DHSS) model. This literature has two important limitations. First, technology is modeled with a constant elasticity of substitu-

*Corresponding author. 
tion (CES) function. ${ }^{1}$ Under a CES specification, input substitutability and essentiality (i.e. an input is essential if positive production requires a positive amount of that input) are fundamentally linked to each other. An elasticity of substitution between the exhaustible resource and capital (which we denote by $\sigma_{k r}$ ) greater than one implies inessentiality of the resource. ${ }^{2}$ Consequently, high substitutability and non-essentiality are confounded and their individual roles in intertemporal sustainability of consumption cannot be identified. Under these technological assumptions the aforementioned studies found that $\sigma_{k r}>1$ is sufficient for existence of a positive sustainable consumption. This framework leaves an important question unanswered: is high substitutability (i.e. $\left.\sigma_{k r}>1\right)$ still sufficient for sustainability when essentiality is preserved?

Moreover, under a CES specification, $\sigma_{k r}<1$ implies bounded average product of the resource as its quantity approaches zero. Consequently, low substitutability and limited resource productivity are also confounded. Under these technological assumptions studies found that $\sigma_{k r}<1$ is sufficient for inexistence of a positive sustainable consumption. But it remains unclear whether limited substitutability (i.e. $\sigma_{k r}<1$ ) is still sufficient for inexistence when average product of the resource is unbounded?

The second limitation is related to technical progress. Previous analyses have been conducted under the assumption of Hicks-neutral technical progress. One exception is [4] who have looked at capital-augmenting technical progress but have not considered resource-augmenting technical progress, nor have they considered neutral and non-neutral technical progress simultaneously. Therefore many questions also remain unanswered pertaining the effect of technical progress on sustainable consumption. Can limited capital-resource substitutability ( $\sigma_{k r}<1$ ) be compensated by technical progress to guarantee existence of a positive consumption path? If so, what kind of technical progress?

We develop a framework capable of 1) linking capital-resource substitutability with the existence of positive sustainable consumption when the resource is strictly essential regardless of the value of $\sigma_{k r}$,2) linking biased technical progress with existence, and 3) capturing compensations between $\sigma_{k r}$ and technical progress that result in existence of a positive sustainable consumption.

\section{Model}

The economy is described by the DHSS model:

$$
\begin{gathered}
\dot{S}_{t}=-r_{t} \\
\dot{K}_{t}=f\left(A, A_{r} r_{t}, A_{k} K_{t}\right)-C_{t}-\lambda K_{t}
\end{gathered}
$$

where $K_{t}$ is the stock of human-made capital at time $t, S_{t}$ is the level of non-renewable resource stock, $r_{t}$ is the flow of the natural resource used in production, $A$ is an efficiency factor capturing Hicks-neutral technological progress, $A_{i}$ is an efficiency factor corresponding to the $i^{\text {th }}$ input $(i=r, K)$ which may increase due to technical progress, $f($.$) is the production function, C_{t}$ is consumption at $t$, and $\lambda$ is the capital depreciation rate. Dots above variables denote time derivatives.

This economy evolves according to the following constraints:

$$
\begin{gathered}
\left\{r_{t} \geq 0\right\}_{t=0}^{\infty} \\
S_{b}=0 \\
\left\{S_{b}<S_{t}\right\}_{t=0}^{\infty} \\
\left\{C_{t}<f\left(A, A_{r} r_{t}, A_{k} K_{t}\right)\right\}_{t=0}^{\infty} \\
\left\{K_{t} \geq 0\right\}_{t=0}^{\infty} \\
\left\{0<C_{b} \leq C_{t}\right\}_{t=0}^{\infty}
\end{gathered}
$$

where $S_{b}$ and $C_{b}$ are arbitrarily chosen values (subscript $b$ denotes "boundary" levels). Henceforth the index $t$

\footnotetext{
${ }^{1}[5]$ uses a generic approximation to the production technology. The downside of this is the impossibility to tie existence conditions to specific technological parameters such as technical progress and elasticity of substitution.

${ }^{2}$ In this situation, as argued by [6], the existence of an exhaustible input is no longer a "fundamental" problem.
} 
will be dropped from the variables for notational simplicity.

The combination of Equations (4) and (5) prevents depletion of the resource in finite time. Equations (2)-(8) can be combined to obtain:

$$
\dot{K} \leq f\left(A, A_{r} r, A_{k} K\right)-C_{b}-\lambda K
$$

We are left with a system of one differential Equation (Equation (1)) and one differential inclusion (Equation (9)) denoting the set of all feasible paths. By solving the system formed by (1) and (9) we find a constant level of the control variable $\left(C_{b}\right)$ that produces trajectories of resource and capital consistent with constraints (2)-(8) at every period $t$. This is, in essence, an intergenerational maximin program where we seek to compute the maximum level of constant consumption. To formally derive conditions for existence of an interterm poral maximin program we employ the "viable control" approach developed by [7] and extended to models of production with exhaustible resources by [4].

Due to the aforementioned limitations of a CES specification we approximate production technology by the following variable elasticity of substitution specification:

$$
Y=A K^{\alpha_{K}+\varepsilon} r^{\alpha_{r}+\gamma} \exp \left(\delta_{k} K^{\frac{\alpha_{K}+\varepsilon}{\alpha_{K}}}\right)
$$

where $A \geq 1, \varepsilon \geq 0, \gamma \geq 0, \alpha_{K}>0$, and $\alpha_{r}>0$.

The function in Equation (10) is a particular case of the more general transcendental function

$$
Y=A K^{\alpha_{K}+\varepsilon} r^{\alpha_{r}+\gamma} \exp \left(\delta_{k} K^{\frac{\alpha_{K}+\varepsilon}{\alpha_{K}}}\right) \exp \left(\delta_{r} r^{\frac{\alpha_{r}+\gamma}{\alpha_{r}}}\right)
$$

where $\delta_{r}=0$. Our particular case features all the desired properties (essentiality of inputs and unbounded average product of the resource as its usage approaches zero, regardless of substitutability) while greatly reducing the analytical demands of the problem. Our approximation to technology nests the Cobb-Douglas specification (when $\delta_{k}=0$ ) widely used in the literature, and incorporates Hicks-neutral technical progress $(A)$, capital-augmenting technical progress $\left(A_{k}=K^{\varepsilon}\right)$, and resource-augmenting technical progress $\left(A_{r}=r^{\gamma}\right)$. By approximating technology with a transcendental specification, our analysis: 1) nests many results in previous studies, 2) examines the robustness of others, and 3) generates new insights regarding the role of substitutability and technical progress on existence of positive sustainable consumption.

\section{Analysis}

We first examine sufficiency of $\sigma_{k r}>1$ on existence of positive sustainable consumption. Our result is summarized in the following proposition.

Proposition 1. Let the DHSS economy be constrained by production function (10). If $\sigma_{k r}>1$ and average product of the natural resource is unbounded (i.e. $\alpha_{r}+\gamma<1$ ), there exists a positive consumption path $\left\{c_{b}>0, r_{t}, K_{t}, t\right\}_{t=0}^{\infty}$ in spite of the resource being essential and regardless of the rate of capital depreciation $(\lambda)$. See proof in Appendix A.

Corollary 1. Positive technical progress is not necessary for existence despite essentiality and capital depreciation. Proof: the conditions $\sigma_{k r}>1$ and $\alpha_{r}+\gamma<1$ guarantee existence even when $\varepsilon=\gamma=0$, and $A=1$.

Corollary 2. Resource-augmenting technical progress can prevent existence. Proof: while the integral in inequality (A.7), Appendix A, converges due to $\delta_{k}>0$, the factor

$T=\left[\frac{C_{b} / K_{0}+\lambda}{1-\left(\alpha_{r}+\gamma\right)}\right]^{\frac{1-\left(\alpha_{r}+\gamma\right)}{\left(\alpha_{r}+\gamma\right)}} \frac{\delta_{k}}{\left(\alpha_{r}+\gamma\right)}{\frac{-1-\left(\alpha_{r}+\gamma\right)-\left(\alpha_{K}+\varepsilon\right)}{\left(\alpha_{r}+\gamma\right)} \alpha_{K}}_{\left(\alpha_{K}+\varepsilon\right)}$ approaches infinity as $\gamma$ approaches $1-\alpha_{r}$.

We prove in Appendix B that our framework replicates sustainability conditions obtained with a Cobb Douglas approximation by [4] in the case of positive capital depreciation (Proposition B.1), and by [2] under zero capital depreciation (Proposition B.2).

The analysis in [2] found that, under a CES specification, $\sigma_{k r}<1$ is sufficient for inexistence of positive sustainable consumption. He reasoned ([2], pp. 34) that, in this case, inexistence is due to the fact that the aver- 
age product of the resource is bounded as the quantity of the resource approaches zero. We revisit this result when a capital-resource elasticity of substitution lower than one and unbounded average product of inputs (i.e. $\lim _{r \rightarrow 0} \frac{y}{r}=\infty$ ) are allowed to coexist.

Proposition 2. Let the DHSS economy be constrained by production function (10) and let $\sigma_{k r}<1$ (i.e. $\left.\delta_{k}<0\right)$. A positive sustainable consumption exists, in spite of the exhaustible resource being essential and regardless of the rate of capital depreciation $(\lambda)$, if the average product of the natural resources is unbounded (i.e. $\left.\alpha_{r}+\gamma<1\right)$ and if $\left(\alpha_{K}+\varepsilon\right) \geq 1+\left(\alpha_{r}+\gamma\right)\left\{\Phi\left(-\frac{\delta_{k}}{\left(\alpha_{r}+\gamma\right)} K^{\frac{\left(\alpha_{K}+\varepsilon\right)}{\alpha_{K}}}\right)-1\right\} \geq 0$, where $\Phi($.$) is a positive and mo-$ notone function of its argument, and it is greater than one if $\delta_{k}<0$. For proof see Appendix C.

This proposition shows that limited substitutability does not necessarily puts the economy in an unsustainable path. It also underscores the importance of capital-augmenting technical progress, as shown in the following corollary.

Corollary 3. If $\alpha_{K}<1$, then capital-augmenting technical progress is necessary for existence. Proof: this result follows from $\left(\alpha_{K}+\varepsilon\right)>1$, which is implied by $\Phi()>$.1 and the existence condition.

Notice that both capital-augmenting $(\varepsilon)$ and resource-augmenting $(\gamma)$ technical progress have an ambiguous effect on the likelihood of existence. This is because, under our transcendental production function, $\varepsilon$ has a positive effect on capital productivity (captured in the left hand side of the inequality) but a negative effect on substitutability $\sigma_{k r}$. The latter implies an increase in $\Phi($.$) which reduces the likelihood of existence. On the$ other hand, $\gamma$ reduces the average product of the resource as its quantity converges to zero (positive effect on the right hand side) but increases substitutabiltiy $\sigma_{k r}$. The latter implies a decrease in $\Phi($.$) which reduces the$ likelihood of existence.

\section{Discussion}

Studies approximating technology with a CES specification, generated two important predictions. First, $\sigma_{k r}>1$ is sufficient for existence [2]-[4]. Second, $\sigma_{k r}<1$ is sufficient for inexistence [2]. Our analysis reinforces the first prediction in a non-trivial context in which essentiality is always preserved and it reverses the second prediction (Proposition 2) when a variable elasticity of substitution specification is used. ${ }^{3}$

Our analysis confirms that $\lim _{r \rightarrow 0} \frac{y}{r}=\infty$ is necessary for existence. However it reveals that $\sigma_{k r} \geq 1$ is not necessary. The result in Proposition 2 demonstrates that $\sigma_{k r}<1 \quad\left(\delta_{k}<0\right)$ does not necessarily condemn an economy to unsustainability. It reveals that the hurdles of limited substitutability can be overcome with a sufficiently high productivity of capital. Equation (A.4) in Appendix A reveals that Hicks-neutral technical progress is beneficial for sustainability in the sense of reducing the minimum level of the resource required to sustain positive consumption. In contrast, resource-augmenting technical progress $(\gamma)$ has an ambiguous effect on sustainability. Hicks-neutral and resource-augmenting technical progress are neither necessary nor sufficient for existence.

Assuming that $\alpha_{r}+\gamma<1$ (which implies $\lim _{r \rightarrow 0} \frac{y}{r}=\infty$ ) the role of capital-augmenting technical progress as a countervailing force to limited substitutability is conceptually illustrated in Figure 1. An elasticity of substitution greater than one guarantees existence of positive sustainable consumption. When $\sigma_{k r}=1$, capital-augmenting technical progress of $1-\alpha_{k}$ or more, guarantees existence of positive sustainable consumption (Proposition B.1). As $\sigma_{k r}$ drops below one, a larger capital-augmenting technical progress is required for existence of positive sustainable consumption (Proposition 2, and Figure C1). As revealed by the existence condition in Proposition 2, there may not exist a level of $\varepsilon$ capable of guaranteeing existence if its effect on $\Phi($.$) is strong$ enough. In this case, the slope of the plotted curve tends to zero for some positive $\sigma_{k r}$. In turn this effect becomes stronger, the larger $\delta_{k}$ in absolute value.

\footnotetext{
${ }^{3}$ Such a specification permits combination of essentiality with unbounded average product of the resource.
} 


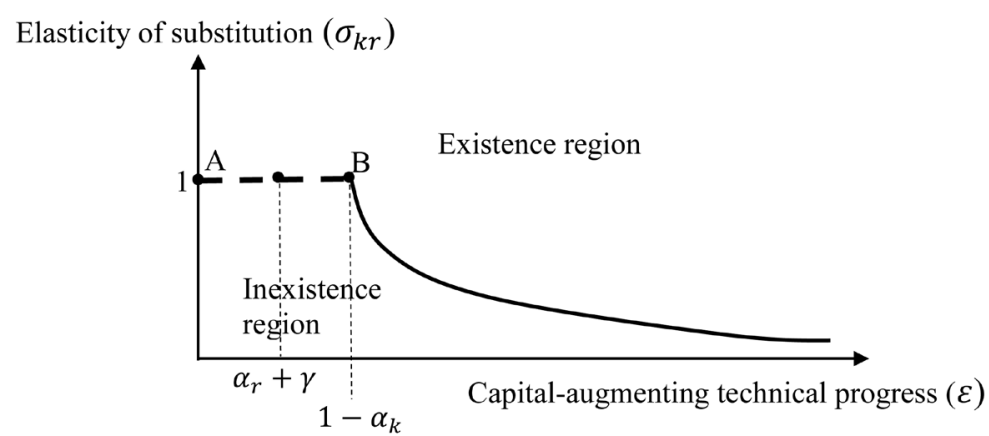

Figure 1. Existence requires higher capital-augmenting technical progress as substitutability decreases.

\section{Conclusions}

This study revisits the issue of existence of positive sustainable consumption in an economy with an essential exhaustible resource. It does so by utilizing a transcendental approximation to technology. Such parametric specification allows formal examination of the effect of capital-resource substitutability while preserving essentiality. We also highlight the role of non-neutral technical progress on existence.

Our analysis offers two important insights. First, $\sigma_{k r}>1$ guarantees existence of positive sustainable consumption even when the exhaustible resource is strictly essential. Second, $\sigma_{k r}<1$ does not necessarily imply inexistence unless it entails $\lim _{r \rightarrow 0} \frac{y}{r}<\infty$ as it is the case with a CES specification. When $\sigma_{k r}<1$, capitalaugmenting technical progress can be an effective countervailing force to limited substitutability, while resource-augmenting and Hicks-neutral technical progress cannot. This issue has not received adequate attention in the literature.

This study extends previous analyses of sustainability under an essential exhaustible resource by considering a particular production function that allows for co-existence of $\sigma_{k r}<1$ and an unbounded average product of the resource. But a general characterization of the family of production functions that preserve these properties while being consistent with stylized facts about growth and allowing decentralization to a market equilibrium is still absent from the literature and constitutes a promising avenue for future research.

\section{References}

[1] Dasgupta, P. and Heal, G. (1974) The Optimal Depletion of Exhaustible Resources. Review of Economic Studies, 41, 1-28. http://dx.doi.org/10.2307/2296369

[2] Solow, R.M. (1974) Intergenerational Equity and Exhaustible Resources. Review of Economic Studies, 41, 9-45. http://dx.doi.org/10.2307/2296370

[3] Stiglitz, J. (1974) Growth with Exhaustible Natural Resources: Efficient and Optimal Growth Paths. Review of Economic Studies, 41, 123-137. http://dx.doi.org/10.2307/2296377

[4] Martinet, V. and Doyen, L. (2007) Sustainability of an Economy with an Exhaustible Resource: A Viable Control Approach. Resource and Energy Economics, 29, 17-39. http://dx.doi.org/10.1016/j.reseneeco.2006.03.003

[5] Mitra, T., Asheim, G.B., Buchholz, W. and Withagen, C. (2013) Characterizing the Sustainability Problem in an Exhaustible Resource Model. Journal of Economic Theory, 148, 2164-2182. http://dx.doi.org/10.1016/j.jet.2013.04.019

[6] Nordhaus, W.D. and Tobin, J. (1972) Is Economic Growth Obsolete. In: Economic Growth, Fiftieth Anniversary Colloquium, V, National Bureau of Economic Research, New York.

[7] Aubin, J.P. (1991) Viability Theory. Springer Verlag, Birkhauser.

[8] Gautschi, W. (1979) A Computational Procedure for Incomplete Gamma Functions. ACM Transactions on Mathematical Software (TOMS), 5, 466-481. http://dx.doi.org/10.1145/355853.355863 


\section{Appendix}

\section{Appendix A}

\section{Proof of Proposition 1.}

The problem at hand is to minimize the natural resource extraction rate subject to technology $f\left(A, A_{k} K, A_{r} r\right)$, differential inclusion (9) and equation of motion (1). To solve this problem we use the viability approach developed by Aubin (1991) and applied to this problem by Martinet and Doyen (2007). Let us define a viability kernel as the set of initial resources and capital levels for which there exists an extraction trajectory that can sustain a positive consumption indefinitely. Let us denote the minimum resource stock for which a positive consumption level can be sustained indefinitely with accumulated capital $K$ by $V(K)$.

Therefore $V(K)$ represents the boundary of the viability kernel which is characterized by an extraction profile that makes $\dot{K}$ and $\dot{S}$ tangent or inward to the viability kernel; i.e. an extraction profile such that $V(K)$ is the solution to the following Hamilton-Jacobi-Bellman (HJB) equation, $\dot{V}+\min _{r \geq r_{b}\left(f, K, C_{b}\right)}\left\{\frac{\partial V}{\partial K} \dot{K}+r\right\}=0$, where $r$ is the flow of the natural resource (to minimize it subject to equations of motion is the primal objective), $r_{b}\left(f, K, C_{b}\right)$ is the minimum resource flow that achieves a consumption level of $C_{b}$ under technology $f\left(A, A_{k} K, A_{r} r\right)$ and capital stock $K$, and $\dot{K}$ is the equation of motion for capital. Assuming capital depreciates at a constant rate of $\lambda$, the time-derivative of capital is $\dot{K}=f\left(A, A_{k} K, A_{r} r\right)-C_{b}-\lambda K$. Plugging the transcendental approximation to technology into the equation of motion yields

$$
\dot{K}=A K^{\alpha_{K}+\varepsilon} r^{\alpha_{r}+\gamma} \exp \left(\delta_{k} K^{\frac{\alpha_{K}+\varepsilon}{\alpha_{K}}}\right)-C_{b}-\lambda K
$$

where $K$ is the capital stock, and $C_{b}$ is constant consumption. Assuming the minimum stock is an autonomous expression (not a direct function of time) implies $\dot{V}=0$. With this information the HJB equation can be re-expressed as:

$$
\min _{r \geq r_{b}(f, K)}\left\{\frac{\partial V}{\partial K}\left[f\left(A, A_{k} K, A_{r} r\right)-C_{b}-\lambda K\right]+r\right\}=0
$$

where $f\left(A, A_{k} K, A_{r} r\right)$ denotes the transcendental technology described in Equation (10).

The first order condition of this problem is:

$$
\frac{\partial V}{\partial K}=-\frac{1}{\frac{\partial f}{\partial r}}
$$

Plugging this back into the HJB equation and solving for the resource flow yields:

$$
r^{*}=\left[\frac{C_{b}+\lambda K}{\left[1-\left(\alpha_{r}+\gamma\right)\right] A} K^{-\left(\alpha_{K}+\varepsilon\right)} \exp \left(-\delta_{k} K^{\frac{\alpha_{K}+\varepsilon}{\alpha_{K}}}\right)\right]^{\frac{1}{\alpha_{r}+\gamma}}
$$

The viability kernel of an economy with accumulated capital $K_{0}$ can be expressed in terms of its partial derivative as $V\left(K_{0}\right)=\int_{0}^{K_{0}} \frac{\partial V}{\partial K} \mathrm{~d} K$ which, since $C_{b}<K_{0}$, can be partitioned and expressed as $V\left(K_{0}\right)=\int_{0}^{C_{b}} \frac{\partial V}{\partial K} \mathrm{~d} K+\int_{C_{b}}^{K_{0}} \frac{\partial V}{\partial K} \mathrm{~d} K$. The first term on the right hand side amounts to $V\left(C_{b}\right)$. We can re-write the viability kernel as $V\left(K_{0}\right)=V\left(C_{b}\right)+C_{C_{b}}+\int_{C_{b}}^{K_{0}} \frac{\partial V}{\partial K} \mathrm{~d} K$, where $C_{C_{b}}$ is a constant of integration. The left hand side is required to converge to the minimum resource requirement $S_{b}$ as capital tends to infinity (in essence, a transversality condition), which results in $S_{b}=\int_{c_{b}}^{\infty} \frac{\partial V}{\partial K} \mathrm{~d} K+V\left(C_{b}\right)+C_{C_{b}}$. Solving this expression for $V\left(C_{b}\right)$ 
and plugging this back into the viability kernel yields $V\left(K_{0}\right)=S_{b}-\int_{C_{b}}^{\infty} \frac{\partial V}{\partial K} \mathrm{~d} K-C_{C_{b}}+C_{C_{b}}+\int_{C_{b}}^{K_{0}} \frac{\partial V}{\partial K} \mathrm{~d} K \quad$ which can be re-expressed as:

$$
V\left(K_{0}\right)=S_{b}-\int_{K_{0}}^{\infty} \frac{\partial V}{\partial K} \mathrm{~d} K
$$

Combining (A.1) and (A.2) and inserting the resulting expression in (A.3) yields:

$$
V\left(K_{0}, C_{b}, S_{b}\right)=S_{b}+A^{-\frac{1}{\alpha_{r}+\gamma}} \int_{K_{0}}^{\infty} K^{-\frac{\left(\alpha_{K}+\varepsilon\right)}{\left(\alpha_{r}+\gamma\right)}}\left[\frac{C_{b}+\lambda K}{1-\left(\alpha_{r}+\gamma\right)}\right]^{\frac{1-\left(\alpha_{r}+\gamma\right)}{\left(\alpha_{r}+\gamma\right)}} \exp \left(-\frac{\delta_{k}}{\left(\alpha_{r}+\gamma\right)} K^{\frac{\left(\alpha_{K}+\varepsilon\right)}{\alpha_{K}}}\right) \mathrm{d} K
$$

An interior path exists if and only if the second term in (A.4) is finite. This occurs whenever the integral converges. We show then that the integral converges if $\delta_{k}>0$.

The integral, which we denote by $I$, can be rearranged in the following way:

$$
I=\int_{K_{0}}^{\infty} K^{\frac{1-\left(\alpha_{r}+\gamma\right)-\left(\alpha_{K}+\varepsilon\right)}{\left(\alpha_{r}+\gamma\right)}}\left[\frac{C_{b} / K+\lambda}{1-\left(\alpha_{r}+\gamma\right)}\right]^{\frac{1-\left(\alpha_{r}+\gamma\right)}{\left(\alpha_{r}+\gamma\right)}} \exp \left(-\frac{\delta_{k}}{\left(\alpha_{r}+\gamma\right)} K^{\frac{\left(\alpha_{K}+\varepsilon\right)}{\alpha_{K}}}\right) \mathrm{d} K
$$

Since the term in brackets depends negatively on the capital stock then the following inequality holds:

$$
I \leq \int_{K_{0}}^{\infty} K^{\frac{1-\left(\alpha_{r}+\gamma\right)-\left(\alpha_{K}+\varepsilon\right)}{\left(\alpha_{r}+\gamma\right)}}\left[\frac{C_{b} / K_{0}+\lambda}{1-\left(\alpha_{r}+\gamma\right)}\right]^{\frac{1-\left(\alpha_{r}+\gamma\right)}{\left(\alpha_{r}+\gamma\right)}} \exp \left(-\frac{\delta_{k}}{\left(\alpha_{r}+\gamma\right)} K^{\frac{\left(\alpha_{K}+\varepsilon\right)}{\alpha_{K}}}\right) \mathrm{d} K
$$

Therefore if the right hand side of inequality (A.6) converges, the left hand side converges. We can re-express the integral in (A.6) as an upper incomplete gamma function. We start with a variable transformation. We first re-define $\frac{\delta_{k}}{\left(\alpha_{r}+\gamma\right)} K^{\frac{\left(\alpha_{K}+\varepsilon\right)}{\alpha_{K}}}$ as variable $x$. Moreover let us transform $K^{\frac{1-\left(\alpha_{r}+\gamma\right)-\left(\alpha_{K}+\varepsilon\right)}{\left(\alpha_{r}+\gamma\right)}}$ to express it in terms of $x$. In particular $K^{\frac{1-\left(\alpha_{r}+\gamma\right)-\left(\alpha_{K}+\varepsilon\right)}{\left(\alpha_{r}+\gamma\right)}}=K^{\left[\frac{1-\left(\alpha_{r}+\gamma\right)-\left(\alpha_{K}+\varepsilon\right)}{\left(\alpha_{r}+\gamma\right)}\right] \frac{\left(\alpha_{K}+\varepsilon\right)}{\alpha_{K}} \frac{\alpha_{K}}{\left(\alpha_{K}+\varepsilon\right)} \frac{\delta_{k}\left\lceil\frac{1-\left(\alpha_{r}+\gamma\right)-\left(\alpha_{K}+\varepsilon\right)}{\left(\alpha_{r}+\gamma\right)}\right] \frac{\alpha_{K}}{\left(\alpha_{K}+\varepsilon\right)}}{\left[1-\left(\alpha_{r}+\gamma\right)-\left(\alpha_{K}+\varepsilon\right)\right] \alpha_{K}}}$. Therefore

$$
\frac{\delta_{k}\left\lceil\frac{1-\left(\alpha_{r}+\gamma\right)-\left(\alpha_{K}+\varepsilon\right)}{\left(\alpha_{r}+\gamma\right)}\right] \frac{\alpha_{K}}{\left(\alpha_{K}+\varepsilon\right)}}{\left(\alpha_{r}+\gamma\right)}
$$

$K^{\frac{1-\left(\alpha_{r}+\gamma\right)-\left(\alpha_{K}+\varepsilon\right)}{\left(\alpha_{r}+\gamma\right)}}$ can be denoted by $x^{\left[\frac{1-\left(\alpha_{r}+\gamma\right)-\left(\alpha_{K}+\varepsilon\right)}{\left(\alpha_{r}+\gamma\right)}\right] \frac{\alpha_{K}}{\left(\alpha_{K}+\varepsilon\right)}} \frac{\delta_{k}}{\left(\alpha_{r}+\gamma\right)}-\left\lceil\frac{1-\left(\alpha_{r}+\gamma\right)-\left(\alpha_{K}+\varepsilon\right)}{\left(\alpha_{r}+\gamma\right)}\right] \frac{\alpha_{K}}{\left(\alpha_{K}+\varepsilon\right)}$. As a result the upper bound of inequality (A.6) is:

$$
I \leq T \int_{F\left(K_{0}\right)}^{\infty} x^{\left[\frac{1-\left(\alpha_{r}+\gamma\right)-\left(\alpha_{K}+\varepsilon\right)}{\left(\alpha_{r}+\gamma\right)}\right] \frac{\alpha_{K}}{\left(\alpha_{K}+\varepsilon\right)}} \exp (-x) \mathrm{d} x=T \Gamma\left[a, F\left(K_{0}\right)\right]
$$

where $\left.T=\left[\frac{C_{b} / K_{0}+\lambda}{1-\left(\alpha_{r}+\gamma\right)}\right]^{\frac{1-\left(\alpha_{r}+\gamma\right)}{\left(\alpha_{r}+\gamma\right)}} \frac{\delta_{k}}{\left(\alpha_{r}+\gamma\right)}-\frac{1-\left(\alpha_{r}+\gamma\right)-\left(\alpha_{K}+\varepsilon\right)}{\left(\alpha_{r}+\gamma\right)}\right] \frac{\alpha_{K}}{\left(\alpha_{K}+\varepsilon\right)}$ and $\Gamma\left[a, F\left(K_{0}\right)\right]$ is the upper incomplete gamma function with $F\left(K_{0}\right)=\frac{\delta_{k}}{\left(\alpha_{r}+\gamma\right)} K_{0}^{\frac{\left(\alpha_{K}+\varepsilon\right)}{\alpha_{K}}}$ as the lower limit of integration and $a=\frac{\left[1-\left(\alpha_{r}+\gamma\right)-\left(\alpha_{K}+\varepsilon\right)\right] \alpha_{K}-\left(\alpha_{r}+\gamma\right)\left(\alpha_{K}+\varepsilon\right)}{\left(\alpha_{r}+\gamma\right)\left(\alpha_{K}+\varepsilon\right)}$. The condition $F\left(K_{0}\right)>0$ is sufficient for absolute convergence of the upper incomplete gamma function [8]. In this case, the condition amounts to $\delta_{k}>0$. We now 
show that this is equivalent to a capital-resource elasticity of substitution higher than one.

The Allen-Uzawa elasticity of substitution between capital and resource is

$$
\sigma_{K r}=\frac{F_{K r}}{F}\left(\frac{\sum_{j} X_{j} f_{j}}{K r}\right)
$$

where $F$ is the determinant of the bordered Hessian of the production function, $f_{j}$ is the marginal productivity of input $j$ ( $j=K, r$ for capital and natural resource respectively), and $F_{K r}$ is the cofactor of $f_{K r}$ in the border Hessian. Replacing determinants, cofactors, and partial derivatives by their corresponding expressions from the transcendental technology and assuming, without loss of generality, that $\gamma=\varepsilon=0$ yields

$$
\sigma_{k r}^{M}=\frac{\alpha_{r} y+\alpha_{K} y+\delta_{k} k y}{2 \alpha_{r}\left[\alpha_{K} \frac{y}{K}+\delta_{k} y\right] k-\alpha_{r} y \frac{\frac{\delta_{k} k+\alpha_{k}}{k}\left(\alpha_{K} \frac{y}{K}+\delta_{k} y\right)-\alpha_{K} \frac{y}{k^{2}}}{\alpha_{K} \frac{y}{K}+\delta_{k} y} k-\left(\alpha_{K} y+\delta_{k} k y\right)\left[\alpha_{r}-1\right]} .
$$

After some algebraic manipulation, this can be re-expressed as $\sigma_{k r}^{M}=\frac{\alpha_{r} y+\alpha_{K} y+\delta_{k} k y}{\alpha_{r} y \frac{\alpha_{K}}{\left[\alpha_{K}+\delta_{k} k\right]}+\alpha_{K} y+\delta_{k} k y}$. It is clear then that:

$$
\sigma_{k r}^{M}=\left\{\begin{array}{l}
\frac{\alpha_{r} y+\alpha_{K} y+\delta_{k} k y}{\alpha_{r} y \frac{\alpha_{K}}{\left[\alpha_{K}+\delta_{k} k\right]}+\alpha_{K} y+\delta_{k} k y}>1 \text { if } \delta_{k}>0 \\
\frac{\alpha_{r} y+\alpha_{K} y+\delta_{k} k y}{\alpha_{r} y \frac{\alpha_{K}}{\left[\alpha_{K}+\delta_{k} k\right]}+\alpha_{K} y+\delta_{k} k y}=1 \text { if } \delta_{k}=0 \text { for all } \alpha_{r} \text { and } y \text { positive. } \\
\frac{\alpha_{r} y+\alpha_{K} y+\delta_{k} k y}{\alpha_{r} y \frac{\alpha_{K}}{\left[\alpha_{K}+\delta_{k} k\right]}+\alpha_{K} y+\delta_{k} k y}<\text { if } \delta_{k}<0
\end{array}\right.
$$

Therefore the condition $\delta_{k}>0$ is equivalent to a capital-resource elasticity of substitution greater than one.

\section{Appendix B}

\section{Proposition B.1.}

If $\delta_{k}=0$ (the Cobb Douglas case) then the boundary of the viability kernel described by Equation (A.4) becomes:

$$
V\left(K_{0}, C_{b}, S_{b}\right)=S_{b}+A^{-\frac{1}{\alpha_{r}+\gamma}} \int_{K_{0}}^{\infty} K^{-\frac{\left(\alpha_{K}+\varepsilon\right)}{\left(\alpha_{r}+\gamma\right)}}\left[\frac{C_{b}+\lambda K}{1-\left(\alpha_{r}+\gamma\right)}\right]^{\frac{1-\left(\alpha_{r}+\gamma\right)}{\left(\alpha_{r}+\gamma\right)}} \mathrm{d} K
$$

Let us denote the integral on the right hand side of (B.1) as $I$. This integral can be re-expressed as $I=\int_{K_{0}}^{\infty} K^{\frac{1-\left(\alpha_{r}+\gamma\right)-\left(\alpha_{K}+\varepsilon\right)}{\left(\alpha_{r}+\gamma\right)}}\left[\frac{C_{b} / K+\lambda}{1-\left(\alpha_{r}+\gamma\right)}\right]^{\frac{1-\left(\alpha_{r}+\gamma\right)}{\left(\alpha_{r}+\gamma\right)}} \mathrm{d} K$. Since the bracketed term in the integrand depends negatively on the capital stock then the following inequality holds:

$$
I \leq \int_{K_{0}}^{\infty} K^{\frac{1-\left(\alpha_{r}+\gamma\right)-\left(\alpha_{K}+\varepsilon\right)}{\left(\alpha_{r}+\gamma\right)}}\left[\frac{C_{b} / K_{0}+\lambda}{1-\left(\alpha_{r}+\gamma\right)}\right]^{\frac{1-\left(\alpha_{r}+\gamma\right)}{\left(\alpha_{r}+\gamma\right)}} \mathrm{d} K
$$


Therefore if the right hand side of the inequality converges, the left hand side converges. Solving the integral on the right hand side of the inequality yields:

$$
I \leq\left[\frac{C_{b} / K_{0}+\lambda}{1-\left(\alpha_{r}+\gamma\right)}\right]^{\frac{1-\left(\alpha_{r}+\gamma\right)}{\left(\alpha_{r}+\gamma\right)}} \frac{\left(\alpha_{r}+\gamma\right)}{1-\left(\alpha_{K}+\varepsilon\right)}\left[\infty^{\frac{1-\left(\alpha_{K}+\varepsilon\right)}{\left(\alpha_{r}+\gamma\right)}}-K_{0}^{\frac{1-\left(\alpha_{K}+\varepsilon\right)}{\left(\alpha_{r}+\gamma\right)}}\right]
$$

The right hand side converges (is a finite number) if and only if $\frac{1-\left(\alpha_{K}+\varepsilon\right)}{\left(\alpha_{r}+\gamma\right)}<0$ or, equivalently, $\left(\alpha_{K}+\varepsilon\right)>1$, which is the result found by Martinet and Doyen (1974).

Proof of Proposition B.2.

If $\delta_{k}=0$ (the Cobb Douglas case), then the boundary of the viability kernel is described by Equation (16). If capital does not depreciate, the boundary of the viability kernel can be expressed as:

$$
V\left(K_{0}, C_{b}, S_{b}\right)=S_{b}+A^{-\frac{1}{\alpha_{r}+\gamma}}\left[\frac{C_{b}}{1-\left(\alpha_{r}+\gamma\right)}\right]^{\frac{1-\left(\alpha_{r}+\gamma\right)}{\left(\alpha_{r}+\gamma\right)}} \int_{K_{0}}^{\infty} K^{-\frac{\left(\alpha_{K}+\varepsilon\right)}{\left(\alpha_{r}+\gamma\right)}} \mathrm{d} K
$$

Solving the integral in (B.4) yields:

$$
V\left(K_{0}, C_{b}, S_{b}\right)=S_{b}+\frac{A^{-\frac{1}{\alpha_{r}+\gamma}}}{\left(\alpha_{r}+\gamma\right)-\left(\alpha_{K}+\varepsilon\right)}\left[\frac{C_{b}}{1-\left(\alpha_{r}+\gamma\right)}\right]^{\frac{1-\left(\alpha_{r}+\gamma\right)}{\left(\alpha_{r}+\gamma\right)}}\left[\infty^{\frac{\left(\alpha_{r}+\gamma\right)-\left(\alpha_{K}+\varepsilon\right)}{\left(\alpha_{r}+\gamma\right)}}-K_{0}^{\frac{\left(\alpha_{r}+\gamma\right)-\left(\alpha_{K}+\varepsilon\right)}{\left(\alpha_{r}+\gamma\right)}}\right]
$$

Therefore the minimum level of resource stock needed to sustain consumption at $C_{b}$ (i.e. $V\left(K_{0}, C_{b}, S_{b}\right)$ ) will be finite if only if $\frac{\left(\alpha_{r}+\gamma\right)-\left(\alpha_{K}+\varepsilon\right)}{\left(\alpha_{r}+\gamma\right)}$ is negative or, equivalently, $\left(\alpha_{K}+\varepsilon\right)>\left(\alpha_{r}+\gamma\right)$, which is the result found by Solow (1974).

\section{Appendix C}

\section{Proof of Proposition 2.}

Existence of a positive sustainable consumption in this case depends upon convergence of the right hand side of inequality (A.6) which, once the constant part is removed from the integral, can be re-expressed as:

$$
I \leq \int_{K_{0}}^{\infty} K^{-\frac{\left(\alpha_{K}+\varepsilon\right)+\left(\alpha_{r}+\gamma\right)-1}{\left(\alpha_{r}+\gamma\right)}} \exp \left(-\frac{\delta_{k}}{\left(\alpha_{r}+\gamma\right)} K^{\frac{\left(\alpha_{K}+\varepsilon\right)}{\alpha_{K}}}\right) \mathrm{d} K
$$

Convergence of the right hand side of (C.1) depends on how fast the integrand converges to zero as $K$ tends to infinity. Since $\delta_{k}<0$, the second factor in the integrand will converge to infinity as $K$ tends to infinity. Therefore the integrand will converge only if the first factor approaches zero fast enough. For a $K_{0}$ arbitrarily close to zero (which yields an upper bound to the boundary of the viability kernel), we can numerically show that the integral converges if and only if

$$
\frac{\left(\alpha_{K}+\varepsilon\right)+\left(\alpha_{r}+\gamma\right)-1}{\left(\alpha_{r}+\gamma\right)} \geq \Phi\left(-\frac{\delta_{k}}{\left(\alpha_{r}+\gamma\right)} K^{\frac{\left(\alpha_{K}+\varepsilon\right)}{\alpha_{K}}}\right)
$$

where $\Phi($.$) is a positive and monotone function of -\frac{\delta_{k}}{\left(\alpha_{r}+\gamma\right)} K^{\frac{\left(\alpha_{K}+\varepsilon\right)}{\alpha_{K}}}$. The convergence condition can be written as an implicit inequality as

$$
\left(\alpha_{K}+\varepsilon\right) \geq 1+\left(\alpha_{r}+\gamma\right)\left[\Phi\left(-\frac{\delta_{k}}{\left(\alpha_{r}+\gamma\right)} K^{\frac{\left(\alpha_{K}+\varepsilon\right)}{\alpha_{K}}}\right)-1\right]:
$$




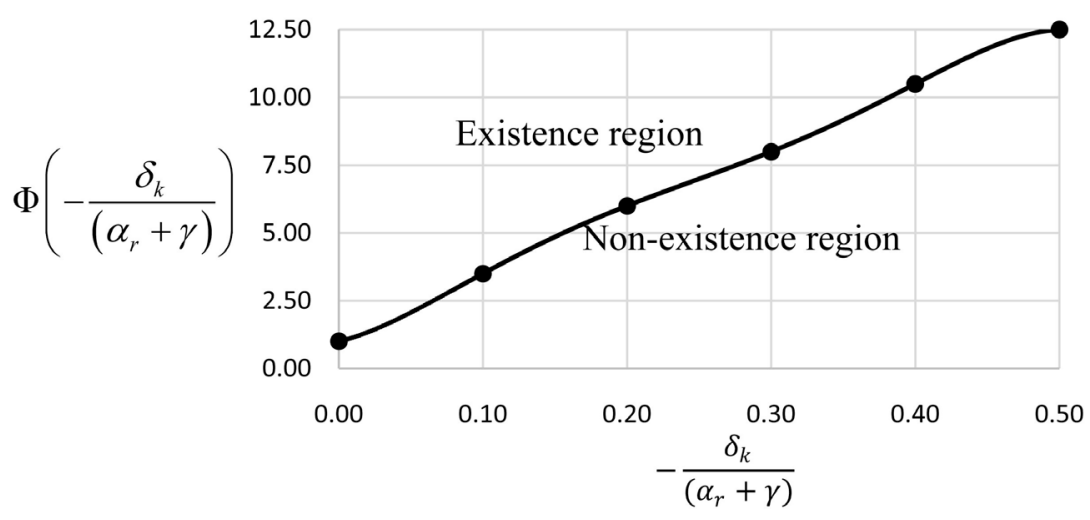

Figure C1. Parametric combinations and existence of sustainable consumption.

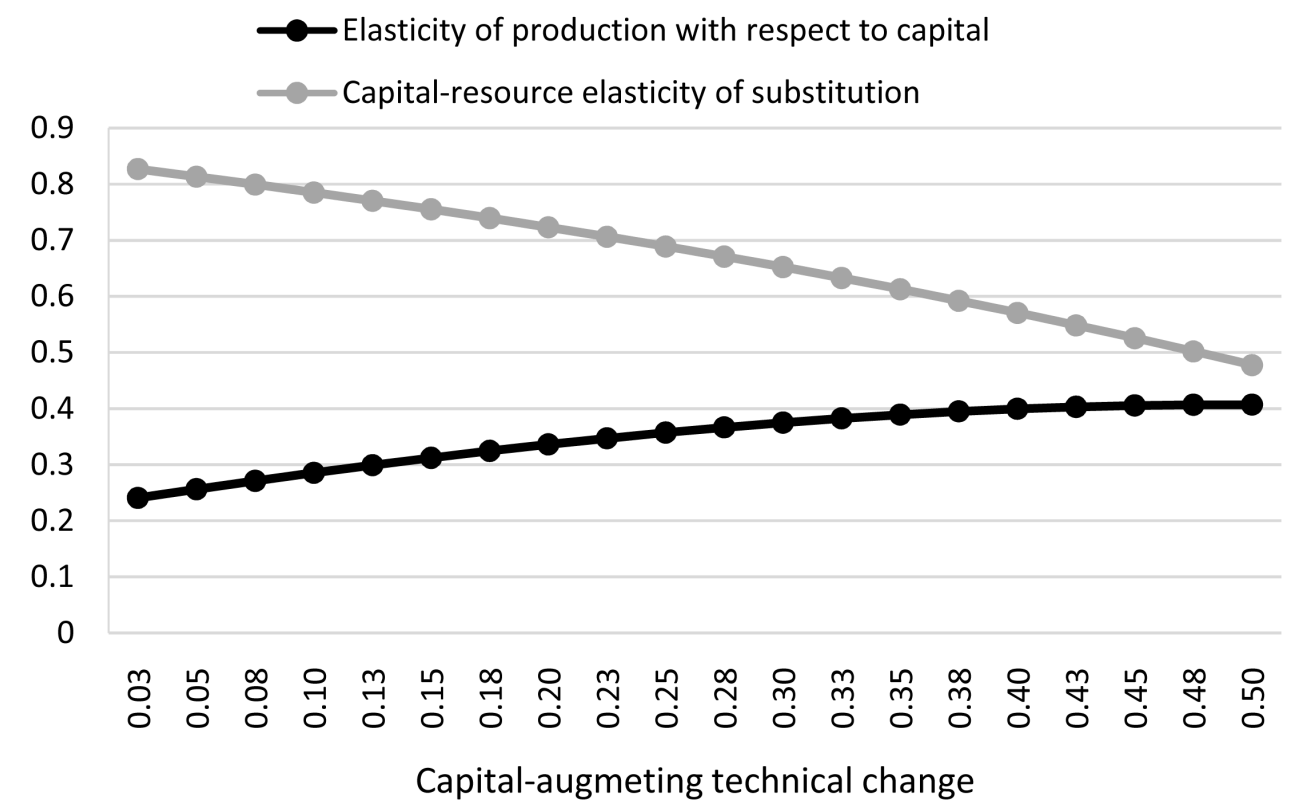

Figure C2. Parametric combinations and existence of sustainable consumption.

We compute the function $\Phi\left(-\frac{\delta_{k}}{\left(\alpha_{r}+\gamma\right)} K^{\frac{\left(\alpha_{K}+\varepsilon\right)}{\alpha_{K}}}\right)$ assuming $K_{0}=1 \mathrm{E}-07, \varepsilon=0$, and $\alpha_{K}=\alpha_{r}+\gamma=0.3$ and depict it in Figure C1. If $\frac{\left(\alpha_{K}+\varepsilon\right)+\left(\alpha_{r}+\gamma\right)-1}{\left(\alpha_{r}+\gamma\right)}$ is higher than the plotted curve for each value of $-\frac{\delta_{k}}{\left(\alpha_{r}+\gamma\right)}$, then there exists a positive sustainable consumption.

Provided $\delta_{k}<0$, the second term on the right hand side of Equation (C.2) is negative. Therefore three facts follow from inequality (C.2). First, $\left(\alpha_{K}+\varepsilon\right)>1$ is necessary for existence (as directly implied by $\Phi()>$.1 ). Therefore, if $\alpha_{K}<1$, capital-augmenting technical change is necessary for existence. Second, resource-augmenting technical change reduces the likelihood of existence and, thus, adversely affects sustainability. Finally capital-augmenting technical change has an ambiguous effect on existence (the left hand side of Equation (C.2) is affected both positively and negatively by $\varepsilon$ ). This is due to the fact that, while increases in $\varepsilon$ raise the marginal productivity of capital (up to a point), they reduce capital-resource substitutability as illustrated in Figure C2 under $K=r=1.5, \alpha_{K}=\alpha_{r}+\gamma=0.3$, and $\delta_{k}=-0.05$. 